\title{
Pressure dependence of the melting mechanism at the limit of overheating in Lennard-Jones crystals
}

\author{
L.Gómez, C. Gazza , H. Dacharry, L. Peñaranda and A. Dobry \\ Facultad de Ciencias Exactas Ingenieria y Agrimensura, Universidad Nacional de Rosario \\ and Instituto de Física Rosario, Avenida Pellegrini 250, 2000 Rosario, Argentina
}

(Dated: November 18, 2018)

\begin{abstract}
We study the pressure dependence of the melting mechanism of a surface free Lennard-Jones crystal by constant pressure Monte Carlo simulation. The difference between the overheating temperature $\left(T_{O H}\right)$ and the thermodynamical melting point $\left(T_{M}\right)$ increase for increasing pressure. When particles move into the repulsive part of the potential the properties at $T_{O H}$ change. There is a crossover pressure where the volume jump becomes pressure-independent. The overheating limit is pre-announced by thermal excitation of big clusters of defects. The temperature zone where the system is dominated by these big clusters of defects increases with increasing pressure. Beyond the crossover pressure we find that excitation of defects and clusters of them start at the same temperature scale related with $T_{O H}$.
\end{abstract}

PACS numbers: 64.70.Dv, 61.72.Ji, 65.40.-b

\section{INTRODUCTION}

Melting is a dramatic phase transition where the translation symmetry present in the solid phase is destroyed at the transition point. As a paradigm of a phase transition in condensed matter physics, its microscopic mechanism has been studied from the beginning of the solid state theory ${ }^{1}$.

Early theories relate melting with an intrinsic elastic instability of the crystal. According to a stability criteria established by Born ${ }^{2}$, melting takes place when an elastic shear modulus vanishes and the crystal looses its ability to resist shear. This was interpreted as due to the softening of a transverse phonon mode with, as a consequence, the homogeneous breakdown of the crystalline order. This notion of crystalline instability refers only to the properties of the crystalline phase but a complete theory of melting should also refer to the properties of the liquid phase.

Therefore, modern theories invoke thermal excitation of extended defects such as dislocation lines 3.4 . Pairs of dislocations can be thermally excited at temperatures within the range of typical solid phases. The presence of dislocations lowers the cost in energy to create an additional dislocation; if the energy reduction is strong enough, it could lead to an avalanche of dislocations resulting in a first order phase transition.

So far, two different ways of melting has been recognized in the literature $\mathrm{e}^{5}$. One related to the thermodynamical melting starting at the surface or extrinsic inhomogeneities of the samples, and the other one with regards to the limit of the mechanical stability of the crystal which takes place when surface is avoided and the solid could be overheated above the equilibrium melting point. Overheating has been experimentally achieved by coating a solid with another material of higher melting point ${ }^{6}$.

As no extrinsic inhomogeneities are present,thermal excitations of defects play an essential role to initiate the melting process. In this work, we will focus on this type of melting.

Although experimental analysis of the microscopic dynamics near melting is very difficult, numerical simulations have recently shown that correlated clusters of defects thermally excited play a central role in this process at the limit of overheating ${ }^{7}$. Moreover in a previous work we have related clusters of defects to dislocation lines assumed in phenomenological theories ${ }^{8}$. However, the situation is far from being solved and continues to puzzle condensed-matter theorists.

An alternative strategy to look for the melting origin could be to change an external parameter and to follow how this parameter affects the melting. The obvious choice of this parameter is the external pressure acting on the solid. Moreover, the pressure dependence of the melting temperature has a practical interest per se and the determination of high pressure melting curves is a subject of many investigations 9.10 .

The purpose of this paper is to discuss the effect of pressure on the melting properties of a surface free crystal by means of a computationally intensive Monte Carlo (MC) calculation using a constant pressure algorithm.

The paper is organized as follows. In Sec. II we analyze some thermodynamic properties at overheating limit. In Sec. III we look at the defect structure preceding the overheating limit. Section IV contains our conclusions and discussion.

\section{PHASE DIAGRAM OF THE LENNARD-JONES SYSTEM}

Our simulations have been performed on a cubic box of 2048 particles ${ }^{11}$ interacting via a Lennard-Jones (LJ) potential written as $V(r)=4 \epsilon\left[\left(\frac{\sigma}{r}\right)^{12}-\left(\frac{\sigma}{r}\right)^{6}\right]$. To guarantee that the potential goes smoothly to zero at distance greater than the cutoff $\left(r_{c}\right)$, a cutoff region from $0.95 r_{c}$ to $r_{c}$ was used following previous work ${ }^{12,13}$. There- 
fore, we can compare our results for the properties at the overheating limit with ones previously obtained at the equilibrium melting point. Moreover we fix the cutoff $r_{c}=2.1 \sigma$ which has been shown to be greater enough to assure convergence of the results $\frac{13}{}$.

The energies are measured in units of $\epsilon$, the distances in units of $\sqrt[3]{4} \sigma$ and the pressure in unit of $\epsilon / 4 \sigma^{3}$. The temperature is reported in units of $\epsilon / k_{B}$. As a concrete example we can take the parameters for argon $(\sigma=3.4 \AA ् \AA, \epsilon=0.0104 \mathrm{eV})$ where our units are: $5.397 \AA$ for the distances, $120.64 \mathrm{~K}$ for the temperatures and $10.5 \times 10^{6} \mathrm{~Pa}$ for the pressures. Our units of pressure differ by a factor $\frac{1}{4}$ from those used in previous works ${ }^{12.13}$. Moreover, our unit of volume differ by a factor 4 .

We emphasized that the use of periodic boundary conditions (PBC) suppresses surface effects and the phase transition temperature we find does not correspond to the thermodynamical melting point but to the limit of overheating of the crystal ${ }^{7}$.

Let us discuss how we constructed the phase diagram of the system. For a fixed pressure at a given temperature, we have averaged the internal energy and the volume over the MC runs. Both the particle coordinates and the volume of the cell are updated in each MC step ${ }^{15}$. Heating is done in a step-by-step procedure starting at low temperature with the particles at the sites of a fcc perfect lattice. The calculation of the jump for different properties (volume, energy, etc.) at the limit of overheating is a difficult task in MC simulation, because of the long CPU time necessary to achieve equilibrium values in this intermediate region. We have densely partitioned the temperature interval and run $\sim 10^{5} \mathrm{MC}$ steps in this zone. In Fig. 1(a) we show a typical results of the evolution of the energy and the atomic volume $(v)$ for $P=1000$ with the temperature. The abrupt jump is related to the limit of overheating. We have observed that both the snapshot of the particle positions as well as the radial distribution function (RDF) above this jump do not show any indication of the crystalline order. By locating the temperature where this jump takes place at different pressures we construct the figure 1(b) where we show the overheating limit $\left(T_{O H}\right)$ as a function of pressure. For comparison, we have included the results for the equilibrium melting point $\left(T_{M}\right)$ extracted from Table II of Ref. 13, obtained by using molecular dynamics (MD) coexistence simulations. We take from this work the results obtained using the same smooth cutoff $r_{c}=2.1 \sigma$ as in our MC simulation.

The two set of values could be fit following the corresponding next laws:

$$
\begin{gathered}
T_{M}=0.036 P^{0.805}+0.66 \\
T_{O H}=0.044 P^{0.806}+0.78
\end{gathered}
$$

Note in figure प(b) that even at $P=0$, the overheating phenomena is observed. While the difference between $T_{M}$ and $T_{O H}$ increase with pressure, the exponent of
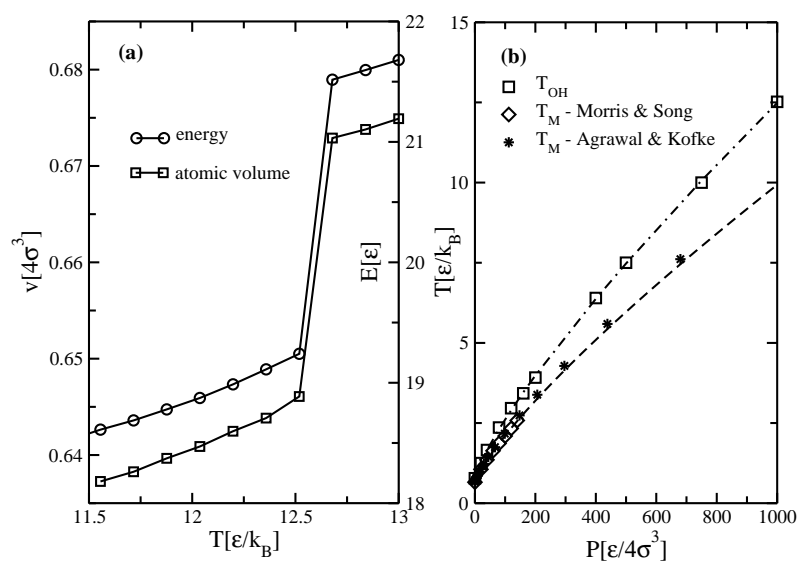

FIG. 1: (a) atomic volume (square, left scale) and internal energy (circles, right scale) vs. the temperature $(T)$ for pressure $P=1000(\mathrm{~b})$ the overheating temperature $\left(T_{O H}\right)$ as a function of the pressure. We also include the melting point $T_{M}$ as obtained in Ref. 13 (open diamond) and from Ref. 12 (starts). Dashed (dot-dashed) lines correspond to the power law fitting given in eq. (1) (eq. (2)) shown in the text.

the pressure is quite similar, $\sim \frac{4}{5}$. In the same figure we have added $T_{M}$ datas (start symbols) from Ref. 12, where the Gibbs-Duhem integration molecular simulation thechnique was used. We can see the agreement with the extrapolation of eq. (11) even in the high pressure regime $\frac{14}{2}$.

Let us analyze this exponent at the light of previous results valid in the regime of high pressure $\frac{16}{\underline{\underline{n}}}$. When particle are close enough, the repulsive part of the potential $\frac{1}{r^{12}}$ dominates and a power law has been deduced $\left(P \beta^{\frac{5}{4}}=\right.$ Const $)$. All this, indicate that the low pressure zone could be neglected in the scale we are presenting the phase diagram.

A microscopic understanding of the differents multiplicative constant shown in the eq. (11) and eq. (2) could be explained with the following argument. While no extrinsic nucleation center as surface or defects are present in our simulation system, in order to break the crystalline order thermal activation of clusters of defects are necessary (see next Section). However, as pressure increase, more thermal energy is needed because particles are strongly compacted. This is different than the process involved in the thermodynamical melting where a liquid phase starts at the surface and pressure is less effective to increase the transition temperature.

The Born criteria has been recently reobtained for homogeneous lattices under an arbitrary but uniform external load ${ }^{17.18}$. In the case of hydrostatic pressure the generalized stability criteria are given by:

$$
\begin{aligned}
B_{T}=\left(C_{11}+2 C_{12}+P\right) / 3 & >0 \\
G=C_{44}-P & >0 \\
G^{\prime}=\left(C_{11}-C_{12}-2 P\right) / 2 & >0
\end{aligned}
$$

where $C_{11}, C_{12}$ and $C_{44}$ are the three different elastic 

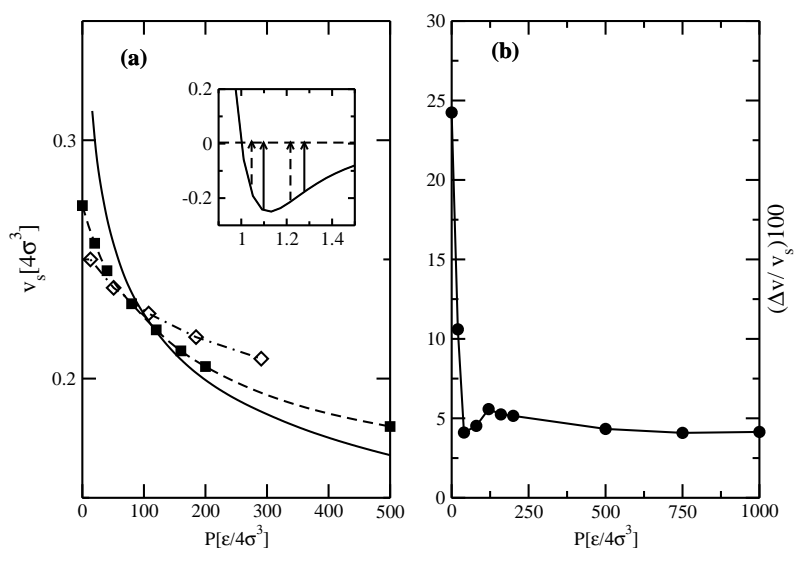

FIG. 2: (a) The atomic volume of the solid $\left(v_{O H}\right)$ at the overheating limit as a function of $P$ (dashed line, square symbols). The solid line shows the volume where $G^{\prime}$ vanishes at $T=0$. We also include the volume at the melting point $\left(v_{M}\right)$ extracted from Ref. 12 (dot dashed line, diamonds). The inset shows the volume dependence of the cohesive energy of an ideal fcc crystal. The dashed (solid) arrow signal the thermal energy to melt (overheat) the crystal. (b) The percentage jump of the atomic volume at the overheating limit.

constants. The $B_{T}, G$ and $G^{\prime}$ are the generalized bulk modulus and the two different shear modulus. We have obtained the elastic constants by means of the harmonic approximation 19 on the Lennard-Jones fcc crystal with interactions up to the third nearest neighbour and variable $v$. This procedure gives the elastic constants at $T=0$ under pure dilation or compression. Note that for a general value of $v$, the crystal is not in equilibrium under the action of the interacting forces and the Cauchy relation $C_{12}=C_{44}$ is not in general satisfied. For each pressure we look for the value of $v$ where some of the module of Eq. vanish. At $P=0, B_{T}$ is the first to be vanished, i.e. for smaller $v$, than the two shear modules. This instability take place at $v=0.315$ higher than the melting detected by MC simulation $(v \sim 0.273)$. From $P>15$ the shear modulus $G^{\prime}$ is the first to become zero. This $v$ signals the volume at $T=0$ where the system could not resist shears. In Fig 2(a) we show these values (solid line) together with the value of $v$ corresponding to the overheating $\left(v_{\mathrm{OH}}\right)$ obtained by MC simulation (square symbols and dashed line).

We have also included in the figure the volume at the melting point $\left(v_{M}\right)$. The density $(\rho)$-temperature $\left(T_{M}\right)$ relationship were extracted from Ref. 12. The specific volume is $v=\frac{1}{\rho}$ and the pressures at melt were obtained from $T_{M}$ using eq. (1).

The three curves intersect at a pressure which separates two regimes. At this pressure, $P_{c} \sim 100, v_{O H}$ is approximately the value where a perfect fcc lattice is in equilibrium under the action of the interacting forces. Therefore the system is dilated at melting for $P<P_{c}$. For $P>P_{c}$, it is compressed. Note that the stability criteria given by Eq.(15) gives an overall correct estimation for the overheating volume. The fact that the vanishing of $G^{\prime}$ shear module gives a good estimation for the superheating volume when solid expands has been recognized in previous works 7.18 .20 . Note however that in a recent study of amorphization under decompression it has been shown that the condition $G^{\prime}=0$ and the softening of a shear phonon signal the critical pressure which destroys the crystalline order ${ }^{21}$, only at $T=0$.

Note in addition, that $v_{M}$ is smaller (greater) than $v_{O H}$ for the dilated (compressed) system. This is consistent with the overheating phenomena found for all pressure. Looking at the inset it is easy to note two different behaviors around the equilibrium volume $\left(v_{e}\right)$. For volumes bellow the $v_{e}$ the thermal energies necessary to destroy a crystal are lower in the overheating case. And in contrary, for volumes above $v_{e}$ the thermal energies are lower in the melting case (see the inset of fig. 2).

The crossover from dilation to compression is also seen in the percentage jump of the atomic volume at the melting $\Delta v / v_{O H}(\Delta v$ is the difference between the liquid and solid volumes per particle). In Fig. 2(b) we show this value obtained from $\mathrm{MC}$ simulation. We can see that for $P>>P_{c}, \Delta v / v_{O H}$ is reduced and becomes almost pressure independent. This behavior could be understood from the relation valid in the high pressure regime. From Eq. (18) of Ref. 13 we see that the specific volume of the solid $\left(v_{S}\right)$ and the liquid $\left(v_{L}\right)$ scale with the same exponent of the temperature:

$$
\begin{aligned}
& 4 v_{L}=C_{1} \beta^{\frac{1}{4}} \\
& 4 v_{S}=C_{2} \beta^{\frac{1}{4}}
\end{aligned}
$$

with $\beta=\frac{1}{k T}, C_{1}=1.23$ and $C_{2}=1.18$. The relative jump of the volume is:

$$
\frac{\Delta v}{v_{s}}=\frac{C_{1}}{C_{2}}-1=0.042
$$

which is $T$ and $P$ independent and of the order of magnitude of the value found in the MC simulation at high pressure as can be seen in Fig. 2 (b)

Microscopically, the structure of the solid near the overheating limit is not the same above and below $P_{c}$. Indeed, the jump percentage of $v$ becomes very small above $P_{c}$ signalling that the structure of the solid below $T_{O H}$ has a lot of defects and could be easily destroyed. We will return to this point in the next section where the evolution of the defect structure will be analyzed.

We now look at the two term of the ClaussiusClapeyron equation obtained from our numerical results. Note that the validity of this equation is not guaranteed a priori because we are calculating the properties at the overheating limit where the free energy of the liquid and solid phase are not equal. It is an interesting point to analyze how different are the properties at $T_{O H}$ from the ones predicted assuming coexistence between the two phases. We have calculated the latent heat of the transition defined as $L_{h}=\Delta u+P \Delta v$, where $\Delta u$ is the jump of the internal energy. In Fig. 3 we compare the two terms 


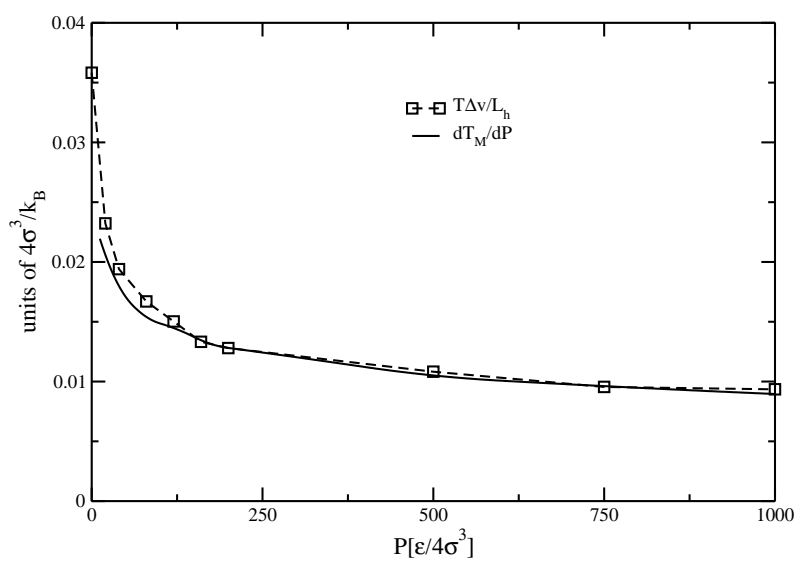

FIG. 3: The two terms of the Clausius-Clapeyron equation evaluated numerically. The solid line corresponds to $\frac{d T_{O H}}{d P}$ evaluated from Eq. 2 Squares correspond to $\frac{T_{O H} \Delta v}{L_{h}}$ where the latent heat $L_{h}$ is obtained from the jumps of the internal energy and the volume $(\Delta v)$.

of the Clausius-Clapeyron equation $T \Delta v / L_{h}$ with $\frac{d T_{O H}}{d P}$ obtained by derivation of the fitting law Eq. 2. Striking the two term coincide at high pressure.

\section{STRUCTURE OF DEFECTS PRECEDING THE OVERHEATING LIMIT}

We have recently found ${ }^{8}$ a premelting temperature at $P=0$ where the number of thermally activated defects increases dramatically and defects start to group in clusters. Near $T_{O H}$ only one cluster across the whole system survives, breaking the crystalline order. In this work we analyze how this feature evolves with the external pressure.

We define a defect as a particle with coordination number $(\mathrm{CN})$ different from 12 , which is the number of nearest neighbors (NN) in an ideal fcc lattice. This CN, called $C_{N N}$, is obtained by counting the number of particles around a given particle up to a cutoff radius. This cutoff is chosen for each pressure as the value where the radial distribution function has its first minimum at low temperature, i.e. the value of the distance between the maxima of the first and the second neighbors.

To compare our results for different pressures we normalize the temperature scale to the value of $T_{O H}$ corresponding to each pressure $\left(\bar{T}=T / T_{O H}\right)$. In Fig. 4 we show the coordination number mean value and the percentage of defects as a function of $\bar{T}$ for different pressures. We see that the reduced temperature where a great number of defects are activated and the crystalline order is perturbed, decreases by increasing pressure. For example for $P=0$ we should go up to $70 \%$ of $T_{O H}$ to have $10 \%$ of defects whereas at $P>P_{c}$ we achieved the same quantity of defects by going only up to $50 \%$ of $T_{O H}$. The decrease of the coordination number shows a similar behavior. These facts are consistent with the small

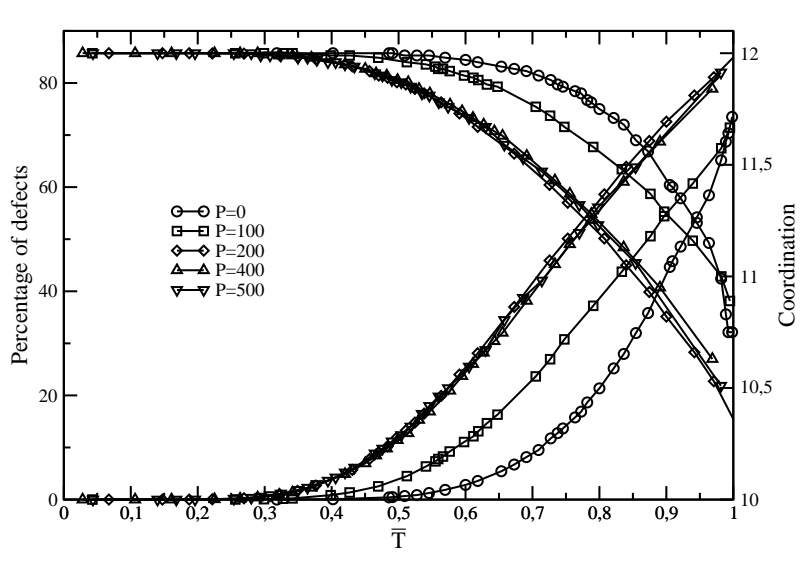

FIG. 4: The percentage of defects (left scale) and the mean coordination number (right scale) as functions of the reduced temperature for different pressures.

jump of the volume between the two phases found in the previous section.

Moreover, it is seen in Fig. 4 that going beyond the crossover pressure $P_{c}$ the curves almost coincide. When the repulsive part of the potential dominates the system could be connected with a hard sphere model with a temperature dependent radius 22 . As we normalize the temperature scale at the $T_{O H}$, the coincidence of the defect structure at high pressure is a manifestation of the equivalence of the models to an effective hard sphere model.

Like we stated in the introduction, modern theories associate the melting process with a proliferation of dislocation lines. This means that defects would have to be correlated near the melting point. To analyze this correlation, we have grouped the defects into clusters by the following methodology. We start from a given defect and search for new defects up to the cutoff distance. For each of these new defects, we undertake the same procedure. We iterate this process up to completion of a cluster of connected defects. Then we take a new defect disconnected to all the previous ones and develop the same procedure. At the end of this process we separate our set of defects in $N_{c l}$ clusters which are disconnected between them. The defects within a cluster are neighbors to each other but they are not connected to the defects of another cluster.

In Fig. [5] we show the mean value of $N_{c l}$ as a function of $\bar{T}$. The decrease of this quantity above a given $\bar{T}$ (called $\left.\bar{T}_{p m}\right)$ should be compared with the increase of the number of defective particles shown in Fig. 4 These facts indicate that the clusters are becoming bigger and that the defects correlate among them for $\bar{T}>\bar{T}_{p m} \cdot \bar{T}_{p m}$ decreases with increasing pressure and becomes constant at high enough pressures. The premelting zone increases as we already emphasized previously. This could be important to detect experimentally excitations of dislocations lines in the study of melting of materials under pressure.

For low pressures we see that the curves move away 


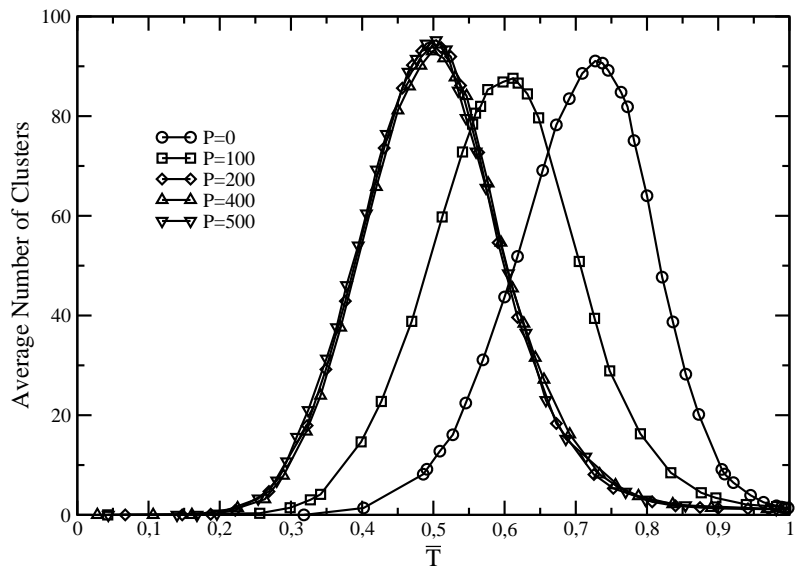

FIG. 5: The average number of clusters as a function of $\bar{T}$ for different pressures.

from the $P=0$ curves and for high pressures they notably coincide with each other. All of these seem to point out that the same mechanism makes the crystal melt at these values of pressure. Note that in a phenomenological theory the crystal melts at a fixed density of dislocation lines at different pressures 23 . At this stage, we cannot give a definitive statement on this prediction but it is suggestive that the tails of all the curves of Fig. 4 correspond to only one cluster. This means that all the defects of the system are interconnected to each other.

Finally, let us notice that Fig. 4 and Fig. 15 show not only the same mechanism of melting at different pressures but also the same structure of thermal excited defects at these pressures.

\section{CONCLUSIONS AND DISCUSION}

We have studied the effect of pressure on the overheating properties of a crystalline solid. We have compared the properties at the overheating limit with the ones at the thermodynamical melting where the solid and liquid could coexist. We have connected the thermodynamic properties with the microscopic dynamics near the overheating limit. We have taken as a representative example an fcc crystal whose particles interact by a Lennard-Jones potential. We have shown that it is possible to obtain equilibrium properties of both phases in the overheating zone by constant pressure MC simulation.

Important conclusions of our study are:

(a) The difference between $T_{O H}$ and $T_{M}$ increase with increasing pressure.

(b) A similar power law fit both the pressure dependence of $T_{O H}$ and $T_{M}$.

(c) Volume expansion due to thermal effects and volume compression due to the external pressure balance at a given pressure. This pressure signal a crossover between two regimes. The volume jump between the two phases becomes rather small( $\sim 5 \%)$ well above the crossover pressure.

(d) The previous result is interpreted at microscopic level as due to the existence of some liquid-like structures in the solid phase. These structures appear as defects in the crystalline phase. Indeed our results show that a great number of defects are activated above a premelting temperature. On the reduced temperature scale, this temperature decreases by increasing pressure and saturates at high pressures.

(d) The premelting temperature also signals the beginning of a temperature range where defects correlate between them grouping in clusters. The number of clusters decreases as the overheating limit approaches from low $T$ by cluster collapsing, leaving only one cluster near the overheating limit.

Our results indicate that experimental detection of premelting behavior, which has been elusive up to now, could be easier detected in high-pressure studies of the melting.

We acknowledge useful discussions with L. Burakovsky and H. T. Diep. We thank A. Belonoshko for sending the datas of his Ref. 14. C.G. thanks to Fundación Antorchas for partial support.
1 For a review, see, e.g. J.G. Dash, Rev. Mod. Phys. 71, 1737 (1999).

${ }^{2}$ M. Born, J. Chem. Phys. 7, 591 (1931).

3 L. Burakovsky, D.L. Preston and R.R. Silbar, Phys. Rev. B 61, 15011 (2000).

${ }^{4}$ F. Lund, Phys. Rev. Lett., 69, 3084 (1992), R. Arias and F. Lund, Defect and Diffusion Forum 150-151, 66 (1997).

${ }^{5}$ R. W. Cahn, Nature (London), 323, 668 (1986).

6 J. Daeges, et. al. Phys. Lett. A 119, 79 (1986)

7 Z. H. Jin, P. Gumbsch, K. Lu and E. Ma, Phys. Rev. Lett. 87, 055703 (2001).

8 L. Gómez, A. Dobry, Ch. Geuting, H. T. Diep, and L. Burakovsky Phys. Rev. Lett. 90, 095701 (2003).

${ }^{9}$ R. Boehler, M.Ross, P. Söderlind and D. B. Boercker,
Phys. Rev. Lett. 865731 (2001).

10 A. B. Belonoshko, S. I. Simak, A. E. Kochetov, B. Johansson, L. Burakovsky, and D. L. Preston, Phys. Rev. Lett. 92, 195701 (2004)

11 We have shown previously ${ }^{8}$ that melting properties do not substantially change by increasing the system size above $N=2048$

12 R. Agrawal and D. Kofke, Mol. Phys. 85, 43 (1995)

13 J. Morris and X. Song, Journ. Ch. Phys. 116, 9352 (2002)

14 A.B. Belonoshko, O. LeBacq, A. Ahuja and B. Johansson, Journ. Ch. Phys. 117, 7233 (2002). In Fig. 4 of this reference the two, MD two-phase and Gibbs-Duhem integration methods, are compared in a great range of pressure.

15 M. P. Allen and D. J. Tildesley, Computer Simulation of 
Liquids, Oxford University Press, Oxford (1987).

16 J. P. Hansen Phys. Rev. A, 2, 221 (1970)

17 J. Wang, S. Yip, S. R. Phillpot and D. Wolf Phys. Rev. Lett. 71, 4182 (1993). J. Wang, Ju Li, S. Yip, S. Phillpot and D. Wolf, Phys. Rev. B 5212627 (1995).

18 J. Wang, Ju Li, S. Yip, D. Wolf and S. Phillpot, Physica A 240, 396 (1997)

19 P. Brüesch, Phonons: Theory and Experiments I. SpringerVerlag, Berlin Heidelberg New York (1982).
20 V. Sorkin, E. Polturak and J. Adler cond-mat/0304215 (2003).

21 S. Bustingorry and E. A. Jagla, Phys. Rev. B 69, 064110 (2004).

22 J. P. Hansen and L. Verlet, Phys. Rev. 184, 181 (1969)

23 L. Burakovsky, D. L. Preston and R. R. Silbar, Journ. of Appl. Phys. 88, 6294 (2000). 